INRA Prod. Anim., 2009, 22 (1), 11-16

\title{
L'utilisation des drêches de blé en alimentation porcine
}

\author{
P. COZANNET 1,3, Y. PRIMOT ${ }^{2}$, J.-P. MÉTAYER ${ }^{3}$, C. GADY ${ }^{4}$, M. LESSIRE $^{5}$, P.-A. GERAERT ${ }^{4}$, \\ L. LE TUTOUR ${ }^{2}$, F. SKIBA', J. NOBLET ${ }^{1}$
}

IINRA, Agrocampus, UMR1079 Systèmes d'Elevage, Nutrition Animale et Humaine, F-35590 Saint-Gilles, France

2 Ajinomoto Eurolysine SAS, 153, rue de Courcelles, F-75817 Paris, France

${ }_{3}^{3}$ Arvalis, Institut du végétal, Boigneville, F-91720 Boigneville, France

${ }^{4}$ Adisseo, France SAS, 42 Avenue Aristide Briand, F-92160 Antony, France

${ }^{5}$ INRA, UR83 Recherches Avicoles, F-37380 Nouzilly, France

${ }^{6}$ Arvalis, Institut du végétal, 21 chemin de Pau, F-64121 Montardon, France

Courriel :Jean.Noblet@rennes.inra.fr

Face à l'augmentation importante du coût des matières premières, l'utilisation optimale de l'ensemble des produits disponibles pour l'alimentation des animaux d'élevage est impérative. La valorisation des coproduits des filières de biocarburants, dont la disponibilité sur le marché européen de l'alimentation animale augmente, est à ce titre, une alternative intéressante. Derrière ce terme de biocarburants se cachent deux filières principales : la filière biodiesel et la filière bioéthanol. Le biodiesel est issu de l'estérification d'huiles végétales extraites en Europe d'oléagineux (colza ou tournesol) ; les coproduits pour l'alimentation des animaux obtenus lors de ce procédé de fabrication sont le tourteau et le glycérol, bien connus et assez largement utilisés en alimentation animale. Le bioéthanol de 1ère génération est obtenu quant à lui par fermentation de glucose par des levures puis distillation de l'alcool. Ce glucose provient de l'hydrolyse enzymatique de l'amidon des céréales (blé, orge ou triticale en Europe et maïs ou sorgho aux USA) ou du saccharose des betteraves sucrières (Europe) ou de la canne à sucre (Brésil). Les coproduits obtenus suite à cette extraction à partir de céréales sont majoritairement les drêches, aussi appelées DDGS pour «Dried Distillers Grains with Solubles», qui contribuent pour une part importante à la rentabilité de la filière (25 à $30 \%$ du chiffre d'affaire).

Très peu de données sont disponibles sur la valeur nutritionnelle de ces produits, notamment pour les drêches de blé qui intéressent plus spécifiquement le marché européen. Les données beaucoup plus nombreuses sur les drêches de maïs, partiellement extrapolables aux drêches de blé, indiquent que ces coproduits ont des compositions chimiques et des propriétés physiques extrêmement variables selon les technologies mises en œuvre (Spiehs et al 2002) et donc des valeurs nutritionnelles potentiellement très variables. L'utilisation des drêches de blé (et des autres céréales à paille) dans l'alimentation des espèces monogastriques (porcs et volailles) passe donc nécessairement par une caractérisation précise des produits afin de définir clairement leurs atouts et leurs contraintes.

\section{1 / Les étapes principales du procédé de fabrication et leurs implications sur la qualité des drêches de blé}

Les procédés de fabrication d'éthanol sont adaptés des procédés permettant la production d'alcool de bouche qui fournissent également des coproduits dénommés drêches. Cependant, afin de répondre aux objectifs de production fixés au niveau européen impliquant d'incorporer 5,75\% de produits d'origine végétale dans les carburants (soit un objectif de production de 11 millions de tonnes de biocarburant en 2010), ils ont été optimisés par l'ajout notamment d'enzymes fibrolytiques interdites dans le cadre d'une production d'alcool alimentaire et industrialisés afin de pouvoir recevoir différents types de substrats en fonction du coût des matières premières.

Le procédé industriel mis en place pour traiter les céréales débute par un broyage de la matière première puis par une mise en solution (empattage) dans l'eau. Des enzymes sont également ajoutées pour hydrolyser l'amidon et les fibres (cellulose) en dextrines puis en glucose. Le glucose ainsi obtenu est ensuite fermenté par des levures (Saccharomyces cerevisiae) qui le transforment en éthanol, dioxyde de carbone et énergie. L'alcool est ensuite distillé afin de séparer l'éthanol de la phase aqueuse et des particules solides résiduelles. La fraction restante est centrifugée, permettant la séparation suivant un gradient de poids de deux fractions : une fraction insoluble ou «Distillers Grains» (DG) appelés aussi grains épuisés et une fraction soluble ou «Distillers Solubles» (DS). Ces deux fractions sont mélangées selon des ratios différents d'une usine à l'autre et séchées permettant d'aboutir au produit terminal, le «Dried Distillers Grains with Solubles». La forme prédominante des produits commercialisés est le DDGS qui représente aux EtatsUnis $60 \%$ des coproduits de production d'éthanol (Shurson et al 2005).

Les étapes principales, présentées cidessus, sont conservées d'une usine à l'autre. Mais des modifications existent dans le traitement des produits à leur réception et dans le traitement des coproduits après l'étape de distillation. On distingue ainsi classiquement deux principaux types de procédés de traitement des graines avant l'empattage qui séparent de façon plus ou moins importante les différentes fractions du grain. Il s'agit soit :

- d'un broyage simple de la graine dans sa totalité (procédé 1),

- de la séparation des enveloppes et de la farine après broyage (procédé 2).

Les modifications apportées à la fin du procédé lors de la phase de traite- 
ment des coproduits portent sur les ratios de mélange entre $\mathrm{DG}$ et $\mathrm{DS}$, le choix du mode et de l'intensité de chauffage et le choix de granuler ou pas le produit. Ces choix techniques couplés aux différentes qualités de matières premières traitées influent grandement sur la variabilité des caractéristiques des drêches obtenues, ce qui constitue un frein majeur à leur emploi dans les aliments pour les espèces monogastriques. Ces sources potentielles de variabilité de composition du coproduit peuvent être classées en deux catégories qui sont 1) la variabilité de la matière première utilisée pour produire de l'éthanol et 2) la variabilité liée au procédé de fabrication lui-même (Knott et al 2004, Singh et al 2007).

\section{2 / Composition chimique, caractéristiques physiques et valeur nutritionnelle pour le porc des drêches de blé}

\section{1 / Composition chimique}

La composition des drêches reflète la composition de la céréale ou du mélange céréalier utilisé dont les résidus, après transformation de l'amidon en éthanol, représentent $96 \%$ de la matière sèche (MS) des drêches, les 4\% complémentaires provenant des ajouts exogènes (levures notamment) lors du procédé d'extraction (Ingledew 1993). Le retrait de l'amidon au cours du processus de production d'éthanol laisse un faible pourcentage d'amidon résiduel

Tableau 2. Valeur énergétique des drêches de blé chez le porc (compilation de données bibliographiques).

\begin{tabular}{|c|c|c|c|c|}
\hline \multirow{2}{*}{ Matières premières } & \multirow{2}{*}{ Blé $^{\mathrm{a}}$} & \multicolumn{3}{|c|}{ Drêches } \\
\hline & & Finlande $^{b}$ & France $^{c}$ & Canada $^{a}$ \\
\hline Composition chimique, $\%$ de MS & & & & \\
\hline Matières azotées & 13,3 & 41,5 & 32,5 & 40,7 \\
\hline Energie brute, MJ/kg MS & 16,9 & 21,4 & 20,4 & 20,4 \\
\hline NDF & 11,8 & 40,9 & 28.6 & 28,6 \\
\hline ADF & 4,8 & 18,2 & 7,2 & 11,5 \\
\hline Digestibilité de l'énergie, $\%$ & 86,2 & 51,7 & 78,1 & 66,6 \\
\hline Teneur en énergie digestible, MJ/kg de MS & 14,6 & 12,8 & 16,0 & 13,6 \\
\hline
\end{tabular}

\footnotetext{
a Nyachoti et al $2005(n=2)$.

b Nasi $1985(n=1)$.

c Jondreville et al $1992(n=1)$.
}

au niveau des coproduits (tableau 1). à l'opposé, les autres constituants, comme les matières grasses et les matières azotées, sont concentrés (niveau multiplié par 3 en moyenne par rapport au blé) (Mustafa et al 1999, tableau 1). Les teneurs en protéines et en matières grasses varient peu au sein de notre panel d'échantillons (32,7 à $39,2 \% \mathrm{MS}$ et 3,4 à $5,7 \% \mathrm{MS}$, respectivement). Cet effet de concentration est également observable au niveau du contenu en parois végétales dont la teneur est multipliée par 3,5 à 4 par rapport au blé (Sauvant et al 2002). De plus, l'augmentation des teneurs globales en fibres s'accompagne d'une modification du profil des parois végétales puisque les teneurs en Neutral Detergent Fibre (NDF), Acid Detergent Fibre (ADF) et Acid Detergent Lignin (ADL) sont multipliées par respectivement 2, 3 et 4 par rapport au blé.

Tableau 1. Concentration en nutriments de 10 drêches de blé d'origine européenne; comparaison au blé (\% MS ; moyenne et écart-type).

\begin{tabular}{|l|c|ccc|}
\hline \multirow{2}{*}{} & Blé $^{\mathbf{a}}(\boldsymbol{n}=7068)$ & \multicolumn{3}{|c|}{ Drêches de bléb $(n=10)$} \\
\cline { 2 - 5 } & Moyenne & Moyenne & Minimum & Maximum \\
\hline Matière sèche, \% & $86,8 \pm 1,1$ & $92,6 \pm 1,9$ & 89,3 & 94,4 \\
Protéines $(\mathrm{N} \times 6,25)$ & $12,0 \pm 0,9$ & $36,4 \pm 2,4$ & 32,6 & 39,2 \\
Amidon & $69,7 \pm 1,9$ & $4,5 \pm 2,6$ & 2,6 & 10,1 \\
Cellulose brute & $2,5 \pm 0,3$ & $8,3 \pm 1,7$ & 6,1 & 11,4 \\
NDF & $12,4 \pm 1,7$ & $28,2 \pm 3,1$ & 22,8 & 33,0 \\
ADF & $3,1 \pm 0,5$ & $11,5 \pm 2,7$ & 7,5 & 16,8 \\
ADL & $1 \pm 0,3$ & $4,7 \pm 2,5$ & 2,1 & 10,3 \\
Matières Grasses & $1,7 \pm 0,2$ & $4,6 \pm 0,7$ & 3,4 & 5,7 \\
Matières minérales & $1,6 \pm 0,2$ & $5,3 \pm 0,8$ & 4,5 & 6,9 \\
Phosphore & $0,37 \pm 0,03$ & $0,87 \pm 0,06$ & 0,80 & 0,97 \\
Calcium & $0,08 \pm 0,03$ & $0,19 \pm 0,08$ & 0,11 & 0,39 \\
Energie Brute (MJ/kg de MS) & $18,2 \pm 0,3$ & $21,0 \pm 0,3$ & 20,5 & 21,5 \\
\hline
\end{tabular}

a Tables INRA-AFZ, Sauvant et al 2004.

b Produits collectés dans le cadre du projet I3A 2007-2010 (INRA, Adisseo, Ajinomoto Eurolysine SAS et Arvalis-Institut du végétal) (Cozannet et al 2009).
Ces modifications du profil en nutriments des drêches par rapport à la céréale originelle rendent les drêches intéressantes pour leur contenu en protéines mais aussi en énergie brute. Toutefois, des variations importantes de ces concentrations existent selon l'origine de l'échantillon, notamment pour la teneur en parois végétales qui, avec l'amidon, sont à prendre en compte pour la différenciation des produits. Les concentrations en amidon et cellulose brute apparaissent ainsi très différentes entre les produits issus du procédé 1 (respectivement 2,4 et $8,6 \%$ de la MS en moyenne) et ceux issus du procédé 2 (respectivement 8,3 et $6,3 \%$ de la MS en moyenne) (Cozannet et al 2009).

\section{2 / Valeur énergétique pour le porc}

La digestibilité de l'énergie contenue dans les drêches est fortement diminuée par une teneur élevée en parois végétales. Ainsi, le coefficient de digestibilité de l'énergie $(\mathrm{dE}, \%)$ des drêches est plus faible que celui du blé (Näsi 1985, Jondreville et al 1992, Nyachoty et al 2005, tableau 2). Toutefois, compte tenu de leur teneur plus élevée en matières grasses et en énergie brute, les teneurs moyennes en Energie Digestible (ED) des drêches sont comparables à celle du blé. Il faut également noter que les valeurs rapportées par ces auteurs sont très variables (de 12,8 à $16,0 \mathrm{MJ} / \mathrm{kg}$ ), illustrant la difficulté de se référer pour ces produits à des données moyennes de référence. Une part importante des différences observées peut être expliquée par les différences de concentration en fibres en relation avec les procédés de fabrication mis en œuvre $(8,3 \%$ d' ADF pour un produit issu du procédé 2 et $12,3 \%$ d'ADF pour deux drêches obtenues par le procédé 1) (Cozannet et al 2009). 
Tableau 3. Profil en acides aminés essentiels de 17 drêches de blés d'origine européenne ; comparaison au blé (\% de $N \times 6,25)$.

\begin{tabular}{|l|c|ccc|}
\hline \multirow{2}{*}{ Sources } & Bléa $^{\mathbf{a}}(\boldsymbol{n = 1 5 0 )}$ & \multicolumn{3}{|c|}{ Drêches de bléb $(n=17)$} \\
\cline { 2 - 5 } & Moyenne & Moyenne & Minimum & Maximum \\
\hline Arginine & $5,10 \pm 0,52$ & $3,81 \pm 0,89$ & 2,25 & 4,64 \\
Cystéine & $2,50 \pm 0,25$ & $1,74 \pm 0,32$ & 0,75 & 2,02 \\
Histidine & $2,34 \pm 0,38$ & $1,97 \pm 0,17$ & 1,66 & 2,22 \\
Isoleucine & $3,60 \pm 0,25$ & $3,43 \pm 0,09$ & 3,28 & 3,63 \\
Leucine & $6,49 \pm 0,41$ & $6,40 \pm 0,26$ & 5,83 & 6,77 \\
Lysine & $2,90 \pm 0,25$ & $2,03 \pm 0,69$ & 0,83 & 3,01 \\
Méthionine & $1,60 \pm 0,17$ & $1,43 \pm 0,07$ & 1,31 & 1,56 \\
Phénylalanine & $4,43 \pm 0,35$ & $4,43 \pm 0,14$ & 4,21 & 4,65 \\
Thréonine & $3,10 \pm 0,24$ & $2,96 \pm 0,13$ & 2,68 & 3,14 \\
Tryptophane & $1,20 \pm 0,13$ & $1,06 \pm 0,11$ & 0,85 & 1,25 \\
Valine & $4,40 \pm 0,40$ & $4,24 \pm 0,12$ & 3,97 & 4,50 \\
\hline
\end{tabular}

a Tables INRA-AFZ, Sauvant et al 2004.

b Produits collectés dans le cadre du projet I3A 2007-2010 (INRA, Adisseo, Ajinomoto Eurolysine SAS et Arvalis-Institut du végétal) (Cozannet et al 2009).

Tableau 4. Digestibilité iléale standardisée des acides aminés de deux drêches de blé issues de deux process différents.

\begin{tabular}{|c|c|c|c|c|}
\hline & \multicolumn{2}{|c|}{ DDGS $1^{a}$} & \multicolumn{2}{|c|}{ DDGS $2^{b}$} \\
\hline Composition, \% de MS & \multirow{2}{*}{\multicolumn{2}{|c|}{35,1}} & \\
\hline Protéines ( $\mathrm{N} \times 6,25)$ & & & & \\
\hline Cellulose brute & \multicolumn{2}{|c|}{8,5} & \multicolumn{2}{|c|}{6,1} \\
\hline Amidon Ewers & \multicolumn{2}{|c|}{3,0} & \multicolumn{2}{|c|}{11,7} \\
\hline Matières Grasses & \multicolumn{2}{|c|}{6,4} & \multicolumn{2}{|c|}{5,7} \\
\hline & $\begin{array}{c}\text { Digestibilité } \\
\%\end{array}$ & $\begin{array}{c}\text { Teneur } \\
\text { en AA } \\
\text { digestible }\end{array}$ & $\begin{array}{c}\text { Digestibilité } \\
\%\end{array}$ & $\begin{array}{c}\text { Teneur } \\
\text { en AA } \\
\text { digestible }\end{array}$ \\
\hline Arginine & 89 & 1,34 & 84 & 1,18 \\
\hline Cystine & 80 & 0,56 & 81 & 0,54 \\
\hline Histidine & 83 & 0,58 & 79 & 0,55 \\
\hline Isoleucine & 83 & 1,00 & 77 & 0,84 \\
\hline Leucine & 86 & 1,98 & 82 & 1,72 \\
\hline Lysine & 72 & 0,43 & 62 & 0,43 \\
\hline Méthionine & 85 & 0,43 & 81 & 0,41 \\
\hline Phénylalanine & 89 & 1,34 & 85 & 1,19 \\
\hline Thréonine & 80 & 0,88 & 75 & 0,75 \\
\hline Tryptophane & 80 & 0,32 & 78 & 0,31 \\
\hline Valine & 82 & 1,23 & 78 & 1,09 \\
\hline
\end{tabular}

a Process 1 : traitement de l'ensemble du grain.

b Process 2 : retrait des sons avant les étapes de fermentation puis réintroduction au coproduit ensuite.

Source : Vilariño et al 2007.

\section{3 / Teneurs en acides aminés et valeur protéique pour le porc}

Les teneurs et le profil en acides aminés des drêches sont le reflet du profil en acides aminés de la matière première et de celui des protéines microbiennes exogènes qui représentent respectivement 94,7 et $5,3 \%$ du contenu des drêches en matières azotées (Ingledew 1993). La comparaison du profil en acides aminés des drêches et de celui du blé (tableau 3) indique cependant une réduction du contenu du produit en trois acides aminés essentiels, la lysine, la cystéine et l'arginine, accompagnée d'un accroissement de la variabilité de leurs teneurs entre les lots. La concentration en lysine, du fait des réactions de Maillard déjà rapportées pour les drêches de maïs par Cromwell et al (1993), est la plus affectée par ces phénomènes et constitue un critère clé à considérer dans le cadre de l'étude de la valeur nutritionnelle des drêches. Ces réactions touchent la fonction réductrice de la lysine et conduisent à la destruction ou au blocage de celle-ci (Carpenter 1960). Cette observation a conduit Stein (2007) à préconiser de n'utiliser que des drêches de maïs présentant des rapports lysine sur protéines totales supérieures à $2,8 \%$ pour l'alimentation des porcs.

Les valeurs de digestibilité iléale standardisée des acides aminés rapportées dans la littérature (Jondreville et al 1992, Nyachoty et al 2005, Vilariño et al 2007, Lan et al 2008) indiquent une digestibilité iléale standardisée voisine de $80 \%$ pour la plupart des acides aminés. Cependant, la digestibilité de la lysine est plus faible $(61 \%$ en moyenne d'après les données de Vilariño et al 2007 et Lan et al 2008) et surtout plus variable que pour les autres acides aminés (tableau 4). Les digestibilités les plus faibles sont d'ailleurs obtenues avec les produits dont la teneur en lysine des matières azotées est la plus faible, ce qui indique que autant la teneur en lysine que sa digestibilité sont dépendantes du process mis en œuvre, notamment à l'étape du séchage au cours de laquelle se produisent des réactions de Maillard touchant la lysine.

En conséquence, le contenu en lysine digestible des drêches est le critère le plus sensible aux caractéristiques du procédé. Les variations du contenu du produit en lysine digestible sont donc dues à la fois à une destruction partielle de la lysine du produit et à une variabilité de sa digestibilité. Il est donc très important de quantifier la teneur en lysine disponible 
des drêches pour apprécier la valeur protéique des drêches.

\section{4 / Caractéristiques physiques}

En plus de la prise en compte de critères de composition chimique dans l'évaluation de la qualité des drêches, plusieurs critères physiques peuvent être intéressants à considérer. La matière première se présente tout d'abord sous forme de farine présentant un grain non homogène, les particules formant parfois des agrégats de matière qui rendent la manipulation et le stockage de ces produits délicats. Ces problèmes conduisent les industriels à préférer la granulation des produits en granulés qui constitue le format majoritaire des produits (photo 1). La couleur et l'odeur du produit sont d'autre part deux critères de premier ordre fortement corrélés aux mesures de valeur nutritionnelle (Cromwell et al 1993). Les mesures réalisées au chromamètre indiquent ainsi une corrélation positive entre la valeur de luminance des drêches de maïs (noir $=0$, blanc $=100$ ) et leur digestibilité (Fastinger et al 2006). Aucune référence comparable n'existe pour les drêches de blé alors que ce critère de luminance est très variable (photo 1). La valeur moyenne (minmax) prise par ce critère est de 53,9 $(43,3-63,2)$ lors de l'étude de 17 produits collectés en Europe. Des observations identiques peuvent être réalisées pour l'odeur qui passe de légèrement fermentée à brûlée, la première correspondant aux produits de qualité satisfaisante et la seconde à ceux de qualité détériorée. Cependant, aucune mesure objective de ce critère n'existe à ce jour pour les drêches de blé.

\section{5 / Teneur et disponibilité du phosphore}

La teneur en phosphore des drêches est de $0,87 \% \mathrm{MS}$, soit une valeur très supérieure à la teneur du blé $(0,37 \%$ MS, tableau 1). Mais la disponibilité est également améliorée par rapport à celle du blé (53 vs $15 \%$; Widyaratne et Zijlstra 2006). Cette disponibilité relativement élevée serait due à la libération du phosphore phytique lors de la fermentation par des phytases microbiennes (Cromwell 1979). Le contenu en phosphore disponible des drêches est donc élevé : 0,59 vs $0,06 \%$ de la MS pour respectivement les drêches de blé et le blé (Cromwell 1979).

\section{3 / Tolérance des porcs à l'incorporation des drêches dans leurs aliments}

Les études s'attachant à définir par stade physiologique le seuil de tolérance des animaux, peu nombreuses pour

Photo 1. Aspect et caractéristiques physiques de quatre qualités de drêches de blé. (C) INRA
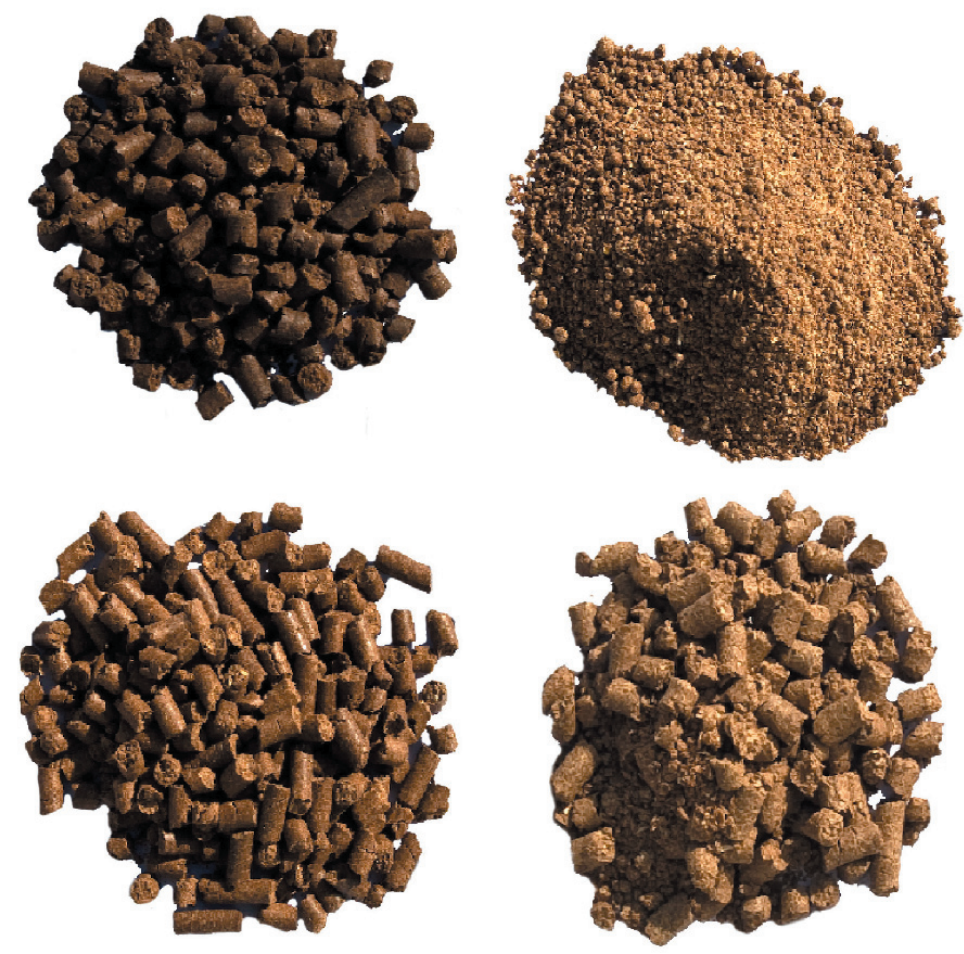

les drêches de blé et plus nombreuses dans le cas des drêches de maïs, donnent des résultats très hétérogènes.

Plusieurs études sur les drêches de maïs et de blé révèlent une réduction de la consommation spontanée, une augmentation de la durée passée à l'auge et une répartition de ce temps passé en un nombre plus important de visites (Whitney et Shurson 2004, Guillou et al 2008). Plusieurs explications ont été apportées à ces phénomènes. Tout d'abord, il pourrait s'agir d'un effet de l'ajout de fibres apportées par la matière première affectant le transit digestif des animaux (Whitney et Shurson 2004, Thacker et al 2006). Ce phénomène est surtout observable chez les animaux les plus jeunes, incapables de valoriser l'excès de fibres apporté par l'ajout de drêches (Govers et al 1999). Mais il peut aussi s'agir d'un déséquilibre entre les acides aminés, en lysine notamment, des régimes incluant des drêches (Henry et al 1992).

Ces effets de l'ajout de drêches dans les régimes impliquent la mise en place de limites d'incorporation afin de minimiser la réduction des performances des animaux. L'analyse de la littérature traitant de l'effet de l'incorporation de drêches dans les régimes pour porcs témoigne de l'importance 1) du stade physiologique de l'animal auquel on destine l'aliment (Whitney et Shurson 2004) et 2) de la qualité intrinsèque du produit (contenu en acides aminés digestibles et en énergie nette notamment) (Cromwell et al 1993, figure 1). Ces observations ont permis de mettre en place des limites basses d'incorporation pour lesquelles aucun effet négatif n'est à craindre quelles que soient les caractéristiques de la drêche. Ces niveaux sont de $10 \%$ pour chacun des stades post-sevrage (Debecker et al 2005) et croissance-finition (Cromwell et al 1984). Des niveaux plus élevés (de 20 à 40\%) (Cromwell et al 1983, Debecker et al 2005) se sont traduits par une dépréciation des performances, notamment chez les animaux les plus jeunes.

\section{Conclusions}

Les drêches de blé représentent une alternative face à la flambée du prix des aliments. Présentant un profil intéressant en tant que source de protéines, d'énergie et de phosphore, ces produits sont utilisables pour l'alimentation des monogastriques, sous réserve cependant de mieux en maîtriser et gérer la 
Figure 1. Influence de l'introduction de drêches dans des aliments sur le gain de poids journalier de porcs en croissance ou en finition ; les performances sont rapportées aux performances du lot témoin sans drêches (compilation des essais de Gralapp et al 2002, Gaines et al 2007, Widmer et al 2008 pour le porc en finition et de Whitney et al 2004, Thacker et al 2006, Barbosa et al 2008, Thomas et al 2008, Widmer et Shurson 2008 pour le porc en croissance ; moyennes corrigées de l'effet essai).

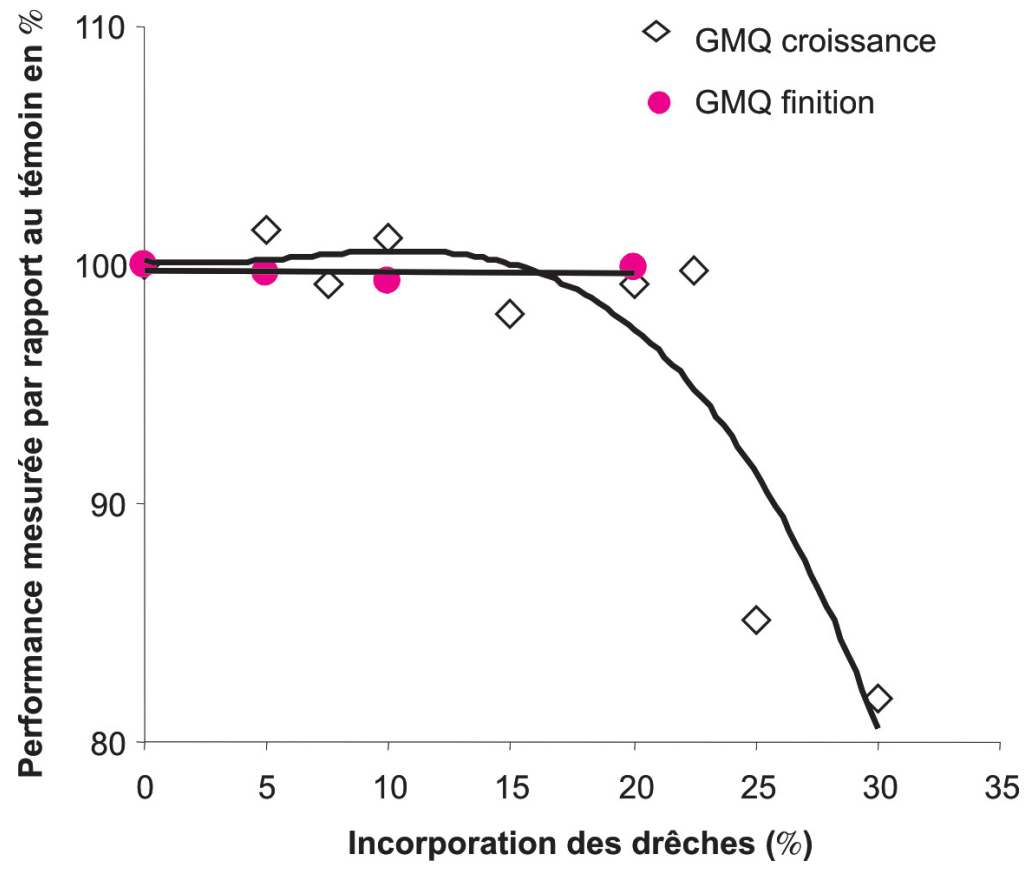

qualité nutritionnelle. Cette maîtrise nécessite une stabilisation des modes de production et de définir les points clefs de variabilité de la composition de ces produits. Le prétraitement des produits et le chauffage du coproduit influencent particulièrement le contenu en fibres et la teneur en lysine ainsi que la digestibilité du produit. La maîtrise de ces étapes permettra de disposer de valeurs plus justes de composition et de digestibilité des produits afin de réaliser une supplémentation adaptée et de ne plus limiter arbitrairement l'incorporation de ces produits dans les régimes. Dans cette optique, un important travail d'évaluation de la digestibilité de l'énergie et des acides aminés est encore nécessaire ainsi que la mise en place d'un ou de systèmes d'évaluation rapide et de prédicteurs de la réponse animale.

\section{Références}

Barbosa F.F., Dritz S.S., Tokach M.D., DeRouchy J.M., Goodband R.D., Nelsen J.L., 2008. Use of distillers dried grains with solubles and soybean hulls in nursery pig diet. J. Anim. Sci., 86 (Suppl. 1), 446.

Carpenter K.J., 1960. The estimation of the available lysine in animal protein foods. Biochem. J., 77, 604-604.

Cozannet P., Primot Y.., Gady C., Métayer J.P., Callu P., Lessire M., Le Tutour L., Geraert P.A., Skiba F., Noblet J., 2009. Valeur nutritionnelle des drêches de blé chez le porc en croissance. Journ. Rech. Porcine Fr., 41, 117-130.

Cromwell G.L., 1979. Availability of phosphorus in feedstuffs for swine. Proc. Distillers Feed Res. Conf., Louisville, KY., 34, 40-52.

Cromwell G.L., Stahly T.S., Monegue H.J., Overfielf J.R., 1983. Distillers dried grain with solubles for growing-finishing swine. Kentucky Agric. Expt. Station. Progress report, 292, Lexington, KY., 30-32.

Cromwell G.L., Stahly T.S., Monegue H.J., 1984. Distillers dried grain with solubles for growingfinishing swine. Kentucky Agric. Expt. Station. Progress report, 284, Lexington, KY., 10-11.

Cromwell G.L., Herkelman K.L., Stahly T.S., 1993. Physical, chemical and nutritional characteristics of distillers dried grains with solubles for chicks and pigs. J. Anim. Sci., 71, 679-686.

DeDecker J.M., Ellis M., Wolter B.F., Spencer J., Webel D.M., Bertelsen C.R., Peterson B.A., 2005. Effect of dietary level of distiller's dried grains with solubles and fat on the growth per- formance of growing pigs. J. Anim. Sci., 83 (suppl. 2), 79.

Fastinger N.D., Latshaw J.D., Mahan D.C., 2006. Amino acid availability and true metabolisable energy content of corn dried distiller grain with solubles in adult cecetomized roosters. Poultry Sci., 85, 1212-1216.

Gaines A.M. Petersen G.I., Spencer J.D., Augspurger N.R., 2007. Use of corn distillers dried grains with solubles (DDGS) in finishing pigs. J. Anim. Sci., 85 (suppl. 2), 96.

Gralapp A.K., Powers W.J., Faust M.A., Bundy D.S., 2002. Effects of dietary ingredients on manure characteristics and odorous emissions from swine. J. Anim. Sci., 80, 1512-1519.

Govers M.J.A.P., Gannon N.J., Dunshea F.R., Gibson P.R., Muir J.G., 1999. Wheat bran affects the site of fermentation of resistant starch and luminal indices related to colon cancer risk: A study in pigs. Gut, 45, 840-847.

Guillou D., Launay C., Landeau E. 2008. Influence de l'incorporation de drêches de bioéthanol dans les aliments sur le comportement alimentaire de porcs nourris ad libitum en croissance-finition. Journ. Rech. Porcine Fr., 40, 215-216.

Henry Y., Colleaux Y., Sève, B. 1992. Effects of dietary level of lysine and o level and source of protein on feed intake, and growth performance, and plasma amino acid pattern in the finishing pig. J. Anim. Sci., 70, 188-195.

Ingledew W.M., 1993. Yeast for production of fuel ethanol. The Yeast $2^{\text {nd }}$ Edition, Yeast
Technology. Academic Press. New York, NY, 5, 245-291.

Jondreville C., Bertin J.M., Grosjean F., 1992. Valeur alimentaire d'un coproduit de la fabrication d'éthanol à partir du blé pour le porc. Journ. Rech. Porcine Fr., 24, 159-166.

Knott J., Shurson J., Goihl J., 2004. Effect of the variability of distiller's solubles and grains within Ethanol plants and the amount of distiller's solubles blended with distiller's grains on fat, protein and phosphorus content of DDGS. Report 2004. 1-5.

Lan Y., Opapeju F.O., Nyachoti C.M., 2008. True ileal protein and amino acid digestibilities in wheat dried distillers' grains with solubles fed to finishing pigs. Anim. Feed Sci. Technol., 140, 155-163.

Mustafa A.F., McKinnon J.J., Christensen D.A., 1999. Chemical characterization and in vitro crude protein degradability of thin stillage derived from barley- and wheat-based ethanol production. Anim. Feed Sci. Technol., 80, 247-256.

Näsi M., 1985. Distillers feeds from various grain as protein sources for pigs J. Agric. Sci. Finland, 57, 255-262.

Nyachoty C.M., House J.D., Slominski B.A., Seddon I.R., 2005. Energy and nutrient digestibilities in wheat dried distillers' grains with solubles fed to growing pigs. J. Food Agric. Sci., 85, 2581-2586.

Sauvant D., Perez J.M., Tran G., 2004. Tables de composition et de valeur nutritive des matières premières destinées aux animaux d'élevage : 
porcs, volailles, bovins, ovins, caprins, lapins, chevaux, poissons. $2^{\text {ème }}$ Edition revue et corrigée. INRA Editions, Paris, France, 301p.

Shurson J., Noll S., Goihl J., 2005, Corn byproduct diversity and feeding value to non-ruminants. $67^{\text {th }}$ Minnesota Nutrition Conf., St. Paul, MN., Sep 19-20, 2006.

Singh V., Parsons C., Pettigrew J., 2007. Process and engineering effect on DDGS products-Present and future. Proc. $5^{\text {th }}$ Mid. Atlantic Nutr. Conf., Baltimore, MD. March 29, 88-90.

Spiehs M.J., Whitney M.H., Shurson G.C., 2002. Nutrient database for distiller's dried grains with solubles produced from new ethanol plants in Minnesota and South Dakota. J. Anim. Sci., $80,2639-2645$
Stein H.H., 2007. Distillers dried grains with solubles (DDGS) in diets fed to swine. Swine Focus. HHS-SwineFocus-001.2007

Thacker P.A., 2006. Nutrient digestibility, performance and carcass traits of growing-finishing pigs fed diets containing dried wheat distiller's grains with solubles. Can. J. of Anim. Sci., 86, 527-529.

Thomas E., Phillip S., Swapna S., Erin E., 2008. Effect of increasing concentrations of distillers dried grains with solubles (DDGS) on growth performance of weanling pigs. Nebraska Swine Report, 2008, 12-13.

Vilariño M., Skiba F., Callu P., 2007. Digestibilité iléale standardisée des protéines et des acides aminés de deux lots de drêches de bioéthanol de blé chez le porc charcutier. Journ. Rech. Porcine Fr., 39, 157-158.
Widmer M.R., McGinnis L.M., Wulf D.M Stein H.H., 2008. Effect of feeding distillers dried grains with solubles, high-protein distillers dried grains, and corn germ to growing-finishing pigs on pig performance, carcass quality, and the palatability of pork. J. Anim. Sci., 86, 1819-1831.

Widyaratne G.P., Zijlstra R. T., 2006 Nutritional value of wheat and corn distiller's dried grain with solubles: digestibility and digestible contents of energy, amino acids and growth performance of grower-finisher pigs. Can. J. Anim. Sci., 87, 103-114.

Whitney M. H., Shurson G. C., 2004. Growth performance of nursery pigs fed diets containing increasing levels of corn distiller's dried grains with solubles originating from moder Midwestern ethanol plant. J. Anim. Sci., 82, 122 128.

\section{Résumé}

Parallèlement à l'accroissement rapide de la demande d'éthanol, essentiellement à base de blé en Europe, la disponibilité pour l'alimentation des animaux d'élevage des coproduits associés, tels que les drêches de blé s'est accrue. La synthèse réalisée dresse le profil moyen de la composition des drêches de blé et indique l'existence d'une forte variabilité de la composition des drêches pour certains paramètres. Ces modifications du profil moyen dépendent des spécificités de traitement du produit. La variabilité est ainsi très importante pour la teneur en parois végétales (NDF) et en amidon dont les teneurs moyennes (min-max) sont respectivement de 28 (23-33) et 4,7 (2,1-10,3)\% de la MS. Les teneurs moyennes en énergie digestible de 14,2 MJ d'ED/kg MS et en phosphore digestible de 0,60\% MS illustrent le potentiel des drêches pour l'alimentation des porcs. Ces valeurs varient selon les sources $(12,8$ à $16,0 \mathrm{MJ} / \mathrm{kg}$ pour l'ED). La teneur en lysine des protéines $(0,83$ à $3,0 \%)$ et la digestibilité iléale standardisée de la lysine $(49$ à $72 \%)$ sont les paramètres les plus variables, vraisemblablement en lien avec le procédé mis en œuvre (séchage notamment). La prise en compte des caractéristiques physiques (odeur et couleur) devrait permettre de prédire la valeur nutritionnelle et de conduire à l'inclusion de drêches à des niveaux plus importants dans l'aliment des porcs.

\section{Abstract}

\section{Wheat Dried Distiller Grains with Solubles for pigs}

In connection with the development of the European ethanol industry from cereals (mainly wheat), co products named dried distiller grains with solubles (DDGS), are more frequently available for animal nutrition. This review proposes the average characteristics of wheat DDGS and indicates that they are highly variable, probably in connection with the preparation of DDGS (drying and associated heating). This effect is especially important for fibre (NDF) and starch with average values (min-max) of 28 (23-33) and 4.7 (2.1-10.3) \% DM, respectively. Average DE content (14.2 MJ per kg DM) and digestible phosphorus (0.60\% DM) illustrate the potential of DDGS in pig diets. However, these nutritional values are highly variable with DE changing according to the fibre level from 12.8 to $16.0 \mathrm{MJ}$ per $\mathrm{kg} \mathrm{DM}$. The lysine content of crude protein $(0.83$ to $3 \%)$ and the ileal digestibility of lysine $(49$ to $72 \%)$ are the most variable characteristics, probably in connection with the process of preparation (i.e., drying). Nevertheless, the inclusion of their physical characteristics (colour and odour) should allow the estimation of the nutritional value of wheat DDGS and contribute to higher rates of inclusion of DDGS in pig diets.

COZANNET P., PRIMOT Y., METAYER J.-P. , GADY C., LESSIRE M., GERAERT P.-A., LE TUTOUR L., SKIBA F., NOBLET J., 2009. L'utilisation des drêches de blé en alimentation porcine. Inra Prod. Anim., 22, 11-16. 